\title{
EL ANÁLISIS FINANCIERO FRENTE A LAS NIIF COMO NUEVA REALIDAD CONTABLE EN COLOMBIA
}

Fabio Puerta Guardo Universidad Tecnológica de Bolívar Colombia

José Vergara Arrieta Universidad Tecnológica de Bolívar Colombia

Leonardo Portela Buelvas Universidad Tecnológica de Bolívar Colombia 
Fabio Puerta Guardo

José Vergara Arrieta

Leonardo Portela Buelvas

\title{
El análisis financiero frente a las NIIF como nueva realidad contable en Colombia
}

\begin{abstract}
Resumen
La presente investigación busca identificar los principios de contabilidad contenidos en el marco contable normativo internacional para Pymes que provocan cambios y ajustes en el análisis financiero. El estudio busca comprender las causas de los cambios en el análisis financiero realizado bajo ratios. Como metodología, se realizó un análisis exhaustivo de las NIIF para las Pymes versión 2015, cuya finalidad consistió en identificar los apartes que inciden en los principios de contabilidad en relación a los consagrados en el marco normativo contable colombiano (esencialmente de los decretos 2649 y 2650 de 1993) y, cómo dichos cambios generan nuevas formas de analizar la información financiera, centrándose en los ratios financieros tradicionales. La revisión evidencia la inexistencia de regulación internacional sobre la aplicación del análisis financiero mediante índices y solo aplicable a aspectos contables. Por otra parte, se ajustan los estados financieros, con la inclusión de nuevas partidas y la libertad de presentación de rubros corrientes y no corrientes, de acuerdo a la naturaleza de las organizaciones. Con los cambios en el marco normativo contable internacional, el análisis financiero mediante ratios se ve afectado, y le corresponde al analista la trazabilidad de los mismos.
\end{abstract}

Palabras clave: Ratios Financieros, NIIF, Pymes, Análisis Financiero

\section{Analyse financière contre les IFRS comme nouvelle réalité comptable en Colombie}

\section{Résumé}

Cette recherche vise à identifier les principes comptables contenus dans le cadre comptable réglementaire international pour les PME qui provoquent des changements et des ajustements dans l'analyse financière. L'étude cherche à comprendre les causes des changements dans l'analyse financière réalisée sous ratios. En tant que méthodologie, une analyse exhaustive de la version IFRS pour les PME 2015 a été réalisée, dont le but était d'identifier les sections qui affectent les principes comptables par rapport à ceux établis dans le cadre réglementaire comptable colombien (essentiellement des décrets 2649 et 2650 1993) et comment ces changements génèrent de nouvelles façons d'analyser l'information financière, en se concentrant sur les ratios financiers traditionnels. La revue met en évidence l'absence de réglementation internationale sur l'application de l'analyse financière par le biais d'indices et uniquement applicable aux aspects comptables. En revanche, les états financiers sont ajustés, avec l'inclusion de nouveaux éléments et la liberté de présenter les éléments courants et non courants, selon la nature des organisations. Avec les changements du cadre réglementaire comptable international, l'analyse financière par les ratios est affectée, et la traçabilité de ceux-ci correspond à l'analyste.

Mots clés: Ratios financiers, IFRS, PME, analyse financière

\section{Financial analysis against IFRS as a new accounting reality in colombia}

\begin{abstract}
This research seeks to identify the accounting principles contained in the international regulatory accounting framework for SMEs that cause changes and adjustments in the financial analysis. The study seeks to understand the causes of the changes in the financial analysis carried out under ratios. As a methodology, an exhaustive analysis of the IFRS for SMEs 2015 version was carried out, the purpose of which was to identify the sections that affect the accounting principles in relation to those established in the Colombian accounting regulatory framework (essentially of decrees 2649 and 2650 1993) and how these changes generate new ways of analyzing financial information, focusing on traditional financial ratios. The review evidences the absence of international regulation on the application of financial analysis through indices and only applicable to accounting aspects. On the other hand, the financial statements are adjusted, with the inclusion of new items and the freedom to present current and non-current items, according to the nature of the organizations. With the changes in the international accounting regulatory framework, the financial analysis through ratios is affected, and the traceability of these corresponds to the analyst.
\end{abstract}

Keywords: Financial Ratios, IFRS, SMEs, Financial Analysis 


\section{El análisis financiero frente a las NIIF como nueva realidad contable en Colombia}

INFORMACIÓN DEL ARTÍCULO

Recepción del artículo: 16/07/2018

Concepto de evaluación: 25/09/2018

Aceptación del artículo: 30/10/2018
Fabio Puerta Guardo* Universidad Tecnológica de Bolívar, Colombia

\author{
José Vergara Arrieta** \\ Universidad Tecnológica de Bolívar, Colombia \\ Leonardo Portela Buelvas \\ Universidad Tecnológica de Bolívar, Colombia
}

\section{INTRODUCCIÓN}

Con la promulgación de la ley 1314 de 2009, Colombia entró oficialmente a la lista de países con obligatoriedad de aplicación de los estándares internacionales de información financiera. La intención del Gobierno de entonces, fue incluir al país en la ruta de la inversión extranjera con la aplicación de técnicas contables y financieras de aceptación internacional. Así, los dueños del capital foráneo podrían fácilmente realizar el análisis e interpretación de la información financiera de empresas nacionales, al manejar un lenguaje contable común al de otras compañías del mundo.

Con fundamento en lo anteriormente expuesto, el presente estudio busca analizar la incidencia en los índices financieros tradicionales con la implementación de las NIIF para las Pymes en Colombia, con el fin de acercar el análisis financiero a la nueva realidad organizacional. Para ello, se analizó minuciosamente la forma en cómo la implementación del marco normativo contable internacional para las Pymes establece modificaciones en los índices financieros, originado en los nuevos conceptos y principios contables que distan de los tradicionalmente aplicados en el país.

En este sentido, el análisis financiero que se desarrolle mediante indicadores, dependerá del tipo de compañía y, por ende, del estándar contable que aplique la misma. Para efectos del presente artículo, el análisis sólo se centró en las NIIF para las Pymes.

\section{REFERENTE TEÓRICO}

Actualmente se establece que "la globalización e internacionalización de las actividades de las empresas; el necesario proceso de armonización y homogeneización internacional de normas y la necesidad de garantizar la trasparencia" (Sánchez, 2006, p.14), ha creado la necesidad de realizar procesos de homogenización y/o estandarización de los principios en el proceso de registro de operaciones financieras y contables (Puerta, Vergara, \& Portela, 2017).

Dicha dinámica conduce a que las empresas no puedan competir si no cuentan con sistemas eficientes y eficaces para la generación de información contable y financiera útil para la oportuna toma de decisiones, tanto para los acreedores como accionistas y demás terceros de un negocio (Horngren, Harrison \& Oliver, 2010; Guajardo \& Andrade, 2008; Warren, Reeve, \& Duchac, 2010; Muñoz, 2008; Meigs, Williams, Haka, \& Bettner, 2000). Al respecto, Muñoz (2008) afirma:

*Email: fpuerta@utb.edu.co

**Autor de correspondencia: jvergara@utb.edu.co 
A consecuencia de la necesidad incuestionable de presentar información financiera confeccionada con criterios uniformes, la profesión contable, los académicos y los organismos más representativos de ésta, inician el proyecto ambicioso de homogeneización contable, con el objetivo de conseguir un mayor grado de comparabilidad de la información ofrecida en los estados financieros. (p.114)

En efecto, "la evolución del mundo de los negocios provoca que los sistemas contables no puedan considerarse cerrados. Ante este constante cambio en la tipología de las transacciones, el regulador señala al Marco Conceptual como la herramienta adecuada para resolver las lagunas regulatorias" (Molina \& Tua, 2010, p.267). Por otro lado:

En el marco actual de la globalización, caracterizada por los tratados de libre comercio, la apertura total de las fronteras y el acceso a los mercados internacionales de capitales, es fundamental la estandarización del lenguaje contable para la elaboración de estados financieros que favorece la transparencia corporativa. En consecuencia, un compromiso estratégico de la comunidad empresarial es hablar el mismo idioma financiero, de ahí la relevancia de la adopción de las Normas Internacionales de Información Financiera (NIIF), pues en más de 100 países de la Unión Europea, América y Asia las han aplicado (Olave, 2013, p.1).

Estas exigencias ocasionaron que un número considerable de países iniciara procesos de convergencia al marco contable normativo internacional. Actualmente se cuenta con perfiles completos para 150 jurisdicciones (IFRS, 2017), tal como se presenta seguidamente:

Tabla 1.

Jurisdicciones que implementan NIIF

\begin{tabular}{|c|c|c|}
\hline & Numero de Jurisdicciones & Porcentaje total \\
\hline Europa & 44 & $29 \%$ \\
\hline África & 23 & $15 \%$ \\
\hline Medio Este & 13 & $9 \%$ \\
\hline Asia y Oceanía & 33 & $22 \%$ \\
\hline Américas & 37 & $25 \%$ \\
\hline Total & 150 & $100 \%$ \\
\hline
\end{tabular}

Fuente: Adaptado de IFRS (2017)

Tabla 2.

Uso de los estándares IFRS en las 150 jurisdicciones perfiladas por región del mundo

\begin{tabular}{|c|c|c|c|c|c|}
\hline Región & $\begin{array}{l}\text { Jurisdicciones } \\
\text { en la región }\end{array}$ & $\begin{array}{l}\text { Jurisdicciones } \\
\text { que requieren } \\
\text { estándares NIIF } \\
\text { para las entidades } \\
\text { nacionales } \\
\text { públicamente } \\
\text { responsables }\end{array}$ & $\begin{array}{l}\text { Jurisdicciones } \\
\text { que requieren } \\
\text { estándares IFRS } \\
\text { ( } \% \text { del total) }\end{array}$ & $\begin{array}{l}\text { Jurisdicciones que } \\
\text { permiten estándares } \\
\text { NIIF para algunas } \\
\text { entidades nacionales } \\
\text { públicamente } \\
\text { responsables }\end{array}$ & $\begin{array}{l}\text { Jurisdicciones que } \\
\text { no requieren } \\
\text { estándares NIIF para } \\
\text { entidades nacionales } \\
\text { públicamente } \\
\text { responsables }\end{array}$ \\
\hline Europa & 44 & 43 & $98 \%$ & 1 & 0 \\
\hline África & 23 & 19 & $83 \%$ & 1 & 3 \\
\hline Medio Este & 13 & 13 & $100 \%$ & 0 & 0 \\
\hline Asia-Oceanía & 33 & 24 & $73 \%$ & 3 & 6 \\
\hline Américas & 37 & 27 & $73 \%$ & 8 & 2 \\
\hline $\begin{array}{l}\text { Totales } \\
\text { omo \% de } 150\end{array}$ & $\begin{array}{c}150 \\
100 \%\end{array}$ & $\begin{array}{l}126 \\
84 \%\end{array}$ & $84 \%$ & $\begin{array}{l}13 \\
9 \%\end{array}$ & $\begin{array}{l}11 \\
7 \%\end{array}$ \\
\hline
\end{tabular}

Fuente: Adaptado de IFRS (2017)

Carneiro, Rodrigues, \& Craig (2017) señalan que "el proceso de armonización contable comenzó a desarrollarse más vigorosamente a medida que los emisores de normas contables regionales y nacionales, y las grandes 4 firmas de auditoría, comenzaron a apoyar al IASB con más fuerza" (p.173). Sin embargo, como lo señala Salazar (2013), "la globalización de los negocios y la nueva arquitectura financiera internacional aceleró la adopción de las NIIF en muchos países" (Salazar, 2013, p.397). Por tanto, la necesidad de comprender y utilizar los distintos estados financieros (domésticos y no domésticos) se incrementó de manera acelerada y proporcional a las actividades de fusión y adquisición internacionales (Choi \& Meek , 2011, p.86). 
Es así que, con fundamento en las exigencias internacionales, inicia la homogenización contable y financiera en el país con la promulgación de la ley 1314 del 2009 (articulo 1) que la acción del Estado debe dirigirse hacia la convergencia de las normas de contabilidad, de información financiera y de aseguramiento de la información, con estándares internacionales de aceptación mundial, implementado las mejores prácticas y conforme a la rápida evolución del sector privado; y el el Ministerio de Comercio, Industria y Turismo clasifico al sector empresarial en grupos (Tabla 3).

Tabla 3.

\section{Grupos para la convergencia NIIF}

\begin{tabular}{|c|c|c|}
\hline Grupos para la convergencia & Decretos reglamentarios & Norma de aplicación \\
\hline $\begin{array}{l}\text { Grupo } 1 \\
\text { a) Emisores de valores; } \\
\text { b) Entidades de interés público; } \\
\text { c) Entidades con planta de personal mayor a } 200 \text { trabajadores o con activos } \\
\text { superiores a } 30.000 \text { SMMLV, y que cumpla con cualquiera de los siguientes } \\
\text { requisitos: } \\
\text {-i. ser subordinada o sucursal de una compañía extranjera que aplique NIIF plenas; } \\
\text {-ii. Ser subordinada o matriz de una compañía nacional que deba aplicar NIIF plenas; } \\
\text {-iii. realizar importaciones o exportaciones que representen más del } 50 \% \text { de las } \\
\text { compras o de las ventas, respectivamente, del año gravable inmediatamente anterior al } \\
\text { ejercicio sobre el que se informa, o } \\
\text {-iv. Ser matriz, asociada o negocio conjunto de una o más entidades extranjeras que } \\
\text { apliquen NIIF plenas. }\end{array}$ & $\begin{array}{l}\text { D. } 2784 \text { de } 2012 \\
\text { D. } 3023 \text { y } 3024 \text { de } 2013\end{array}$ & NIIF Plena \\
\hline $\begin{array}{l}\text { Grupo } 3 \\
\text { Microempresas: } \\
\text { a) Planta de personal no superior a } 10 \text { trabajadores, o } \\
\text { b) Activos totales inferiores a } 500 \text { SMMLV }\end{array}$ & $\begin{array}{l}\text { D. } 2706 \text { de } 2012 \\
\text { D. } 3019 \text { de } 2013\end{array}$ & $\begin{array}{l}\text { Paquete simplificado } \\
\text { de normas contables. }\end{array}$ \\
\hline $\begin{array}{l}\text { Grupo } 2 \\
\text { a) Los que no cumplan con los requisitos para pertenecer al Grupo } 1 \text { o } 3 \\
\text { b) Pequeñas y medianas empresas - Pyme }\end{array}$ & $\begin{array}{l}\text { D. } 3022 \text { de } 2013 \\
\text { D. } 2420 \text { y } 2496 \text { de } 2015\end{array}$ & NIIF Pymes \\
\hline
\end{tabular}

Fuente: Elaboración propia a partir de Deloitte ToucheTohmatsu (2013)

Con el detalle de los decretos expedidos, los periodos de convergencia en Colombia se establecieron entre 2014-2016, con fundamento en la clasificación empresarial, contemplada en la Ley 590 de julio 10 de 2000 , modificada por la Ley 905 de 2004 (artículo $2^{\circ}$ ):

Tabla 4.

Clasificación de las empresas colombianas

\begin{tabular}{l|lll}
\hline $\begin{array}{l}\text { Tipo de } \\
\text { Empresa }\end{array}$ & Empleados & Activos & Activos en pesos colombianos 2017 (SMMLV: \$737.717) \\
\hline Micro & Hasta 10 & Menos 500 SMMLV & $\$ 368.858 .500$ \\
Pequeña & Entre 11 y 50 & Entre 501 y menor de 5.000 SMMLV & Entre \$369.596.217 y menor de \$3.688.585.000. \\
Mediana & Entre 51 y 200 & Entre 5.000 y menor de 30.000 SMMLV & Entre \$3.688.585.000 y menor de \$22.131.510.000.
\end{tabular}

Fuente: Elaboración propia a partir de la Ley 905 de 2004.

El proceso de implementación de las NIIF para el grupo 2 se consideró un año más tarde de haber iniciado en proceso de convergencia con las grandes empresas. Ello, en razón de que gran parte de las empresas nacionales (Pymes) formalizadas con la obligación de llevar contabilidad pertenecen a dicho grupo. Éstas según la Red de Cámaras de Comercio de Colombia - CONFECÁMARAS presentan una participación en la economía colombiana por encima del 99\% del conglomerado nacional, es decir, para el año 2016, las microempresas representaron el 92,1\% del conglomerado nacional, las pequeñas el 5,9\%, las medianas el $1,5 \%$ y las grandes el $0,5 \%$. Ello sin lugar a dudas, refleja la importancia de dicho conglomerado en Colombia (Puerta, Vergara \& Portela, 2017, p.222).

Lo anterior no es un caso atípico a nivel global, dado que la mayoría de las entidades en todo el mundo están representadas por "Pymes", las cuales constituyen más del 95\% de todas las empresas a nivel mundial (Wong, 2004; Alp \& Ustundag, 2009; RSM International Association, 2009). En efecto, el sector de las Pymes se considera la columna vertebral de muchas economías tanto en países del primer mundo como en emergentes. Este sector hace una importante contribución a la creación de empleo, la innovación tecnológica y la 
producción económica. (Chen, 2006; OECD, 2005; Reddy, 2007). Según Sava, Mârza \& Eşanu (2013), "las pequeñas y medianas empresas (PYME) pueden considerarse importantes actores en el bienestar de las comunidades locales y regionales, con un potencial considerable para la creación de empleo" (p.713).

Por lo tanto, ha sido necesario preparar un estándar de contabilidad simplificado y separado para este grupo de empresas (Pymes). El Consejo de Normas Internacionales de Contabilidad (IASB) elaboró un nuevo conjunto de normas denominado "NIIF para Pymes" (Alp \& Ustundag, 2009, p.682). Así las cosas, como resultado de la introducción de las NIIF para las Pymes, en varias jurisdicciones están atrayendo una gran atención en la actualidad y también han entrado en el campo de los informes contables a nivel mundial (Perera \& Chand, 2015).

Es probable que un lenguaje de informes financieros globales eleve la comparabilidad internacional y el reconocimiento mundial de las Pymes en todo el mundo. Por lo tanto, se espera que este cambio sea un gran avance para las Pymes si los beneficios percibidos son debidamente reconocidos por los países y adoptan las NIIF para las Pymes. (p.165) Perera y Chand (2015, p.165). Por otro lado, la importancia de las NIIF radica en posibilitar a este grupo de empresas acceder a los capitales internacionales a través de estados financieros armonizados y de alta calidad (Perera \& Chand, 2015, p.166). Lo anterior se refleja en el hecho que, de las 150 jurisdicciones, 85 requieren o permiten la Norma NIIF para las PYMES, y actualmente está bajo consideración en otras 11 jurisdicciones (IFRS, 2017).

Con la implementación de las NIIF para las Pymes, se presentaron cambios en procesos contables de registro y presentación, algunos sustanciales dependiendo de la partida de los estados financieros a que se haga alusión. La revisión bibliográfica así lo evidencia (Arango \& Torres, 2014; Álvarez, Orozco, \& León, 2013; Salazar, 2011) y se destaca que:

El cambio como consecuencia de la convergencia hacia las NIIF en busca de las mejores técnicas contables y financieras, así como de principios globalmente aceptados para la presentación de estados financieros, implica generar una transformación aplicada a la medición del desempeño corporativo utilizando comúnmente las herramientas de análisis como los ratios financieros (Puerta, Vergara, \& Portela, 2017, p.222).

Tal como expresa Guevara \& Correa (2014):

Con la implementación de las NIIF en Colombia, el procedimiento para reconocer, medir, valorar y revelar los hechos económicos va a cambiar, alterando la forma en que se leen y analizan los estados e indicadores financieros (parte de la planeación financiera y administrativa), y, por ende, la toma de decisiones empresariales. (p.4)

Es el caso de "la elección entre el modelo del valor razonable y el modelo del costo, en la medición de activos no financieros, afecta las ratios de rentabilidad que se obtienen de la información presentada por las entidades" (Castellanos, 2015, p.62). Del mismo modo, "La adopción del modelo normativo IFRS en Colombia genera cambios significativos en lo que atañe a aspectos técnicos. En efecto, el cambio de un modelo normativo basado en reglas a un modelo basado en principios" (Molina \& Tua, 2010, p.264). Lo anterior significa un cambio integral de las empresas, lo que implica que no sólo el equipo contable debe hacer ajustes: si la contabilidad sufre un cambio por este nuevo marco normativo, necesariamente el análisis que se haga de la información contable también debe ajustarse (Puerta, Vergara, \& Portela, 2017, p.223).

En este punto, es preciso destacar la importancia del análisis financiero, definido por García (2015) como:

El examen, comparación, distinción y síntesis de las condiciones, elementos, propiedades y características financieras de la misma. El análisis financiero con un enfoque integral, es la expresión total y completa del análisis financiero, es decir, es un estudio profundo de una organización que considera cada uno de los elementos y características que influyen directa e indirectamente en el desempeño financiero. (p.5)

Por su parte Wild, Subramanyam, \& Halsey (2007), lo definen como "un conjunto de procesos analíticos que forman parte del análisis de negocios. Estos procesos separados tienen algo en común: utilizan la información de los estados financieros, en diversos grados, para propósitos del análisis" (p.13). De esta manera resulta importante el aporte de Según Van Horne \& Machowicz (2010):

El tipo de análisis varía de acuerdo con los intereses específicos de quien lo realiza. Los acreedores (proveedores de dinero para bienes y servicios) están interesados principalmente en la liquidez de la 
empresa. Sus demandas son a corto plazo, y la habilidad de pagar estas demandas con rapidez se juzga mejor mediante el análisis de liquidez de la empresa. (p.128)

Tabla 5.

Cualidades de la información financiera

\begin{tabular}{l|l}
\hline Cualidad & Descripción \\
\hline Calidad & $\begin{array}{l}\text { Trasparencia y rigurosidad de los datos contable } \\
\text { Suficiencia }\end{array}$ \\
$\begin{array}{l}\text { Completa en sus contenidos y recoja toda la información necesaria para interpretar el pasado y hacer predicciones } \\
\text { sobre el futuro incorporando las características de universalidad, utilidad y comparabilidad. }\end{array}$ \\
Homogeneidad & $\begin{array}{l}\text { En la forma de representar los hechos económicos, condición que debe interpretarse tanto en lo que se refiere a } \\
\text { los contenidos como en el tratamiento que realizan las empresas de la información recibida en el tiempo y en el } \\
\text { espacio, incluyendo la eliminación de diferencias que se generan como consecuencia de los distintos usos y } \\
\text { costumbres y normativa aplicable de distintos países o de distintas áreas geográficas. }\end{array}$
\end{tabular}

Fuente: Elaboración propia a partir de Sánchez (2006, p.11)

A pesar de las condiciones que un analista financiero busca en la información contable (Tabla 5), el análisis de los Estados Financieros no tiene una estructura formal dado que carece de definidores, marco conceptual y reguladores y, no está codificada. En éste sentido, "no hay autoridad formal de la que emanen las reglas para el desarrollo del mismo, simplemente hay un conjunto de prácticas y enfoques aceptados" (Entwistle, 2015, p.557). Las herramientas de análisis más comunes y que se aplican constantemente en las empresas son las razones o ratios financieros. Mayes \& Shank (2012) refieren que las razones son el microscopio de un analista; nos permiten tener una mejor imagen de la salud financiera que sólo ver los estados financieros sin pulir. Según Robinson, Van Greuning, Henry, \& Broihahn (2009, p.266):

No hay órganos autorizados que especifiquen fórmulas exactas para computar ratios o proporcionar una lista estándar y completa de relaciones. Fórmulas e incluso nombres de ratios a menudo difieren de analista a analista o de base de datos a base de datos. El número de relaciones diferentes que se pueden crear es prácticamente ilimitado. Sin embargo, hay relaciones ampliamente aceptadas que se han encontrado útiles. (p.266)

Tradicionalmente, el análisis de los estados financieros se ha realizado utilizando un conjunto de relaciones para resaltar el desempeño relativo de una empresa en comparación con su industria. "El número de relaciones que se pueden calcular sobre la base de datos financieros está limitado sólo por la imaginación del analista. Sin embargo, sólo un subconjunto del número potencialmente infinito de relaciones se puede interpretar de manera significativa" (Feroz, Kim \& Raab, 2003, p.49). Ahora bien, según Özkan y Erdener (2010):

El análisis de estados financieros bajo NIIF no sólo está relacionado con herramientas y técnicas analíticas (análisis de ratios, análisis vertical y horizontal, etc.), sino también estrechamente asociado con el análisis de todos los eventos y transacciones financieras y no financieras presentados en los estados financieros Y sus notas. Por lo tanto, el análisis de los informes basados en las NIIF debe ser tan importante como la preparación de los mismos. Sin embargo, la mayoría de los estudios relacionados con las NIIF en la literatura están proporcionando orientación para las necesidades prácticas de los preparadores, en lugar de abordar los temas desde los puntos de vista de los analistas. (Özkan y Erdener, 2010, pág. 50).

Acorde con ello, Janica \& Piñeiro (2008), aseveran que la "contabilidad organizada mediante esquemas internacionales mostrará estados financieros con información de calidad, comparable y coherente con los requerimientos de los usuarios, lo que se convierte en ventaja competitiva al momento de efectuar compensaciones económicas en escenarios nacionales e internacionales". Finalmente, García \& Dueñas (2016) señalan:

Los beneficios de acoger las NIIF, por una parte, permiten que una Pyme presente sus estados financieros en similares condiciones que sus competidores internacionales, aumentando la calidad y comparabilidad de la información financiera, haciendo a los profesionales contables más competentes en cuanto a la utilización de una expresión contable a escala mundial; el estudio de las NIIF incrementará la competitividad de los diferentes sectores económicos. Asimismo, habrá interés de inversión extranjera hacia mercados nacionales, lo cual permitirá toma de decisiones de forma más rápida transparente y hablar un "idioma contable común" entre países, y ello hace que se requiera de una inversión considerable para la implementación de aplicativos adaptados al modelamiento de las políticas contable, de tal manera que se ajusten al nuevo esquema de presentación de los estados financieros. (p.117) 
Las NIIF buscan la homogeneización de las prácticas contables en el mundo a fin de que un lenguaje financiero común dinamice las operaciones entre inversionistas, organizaciones, gobiernos y demás actores, y sobre todo asegurar la calidad de la información (Confetti, Da Silva, Ambrozini, \& Guasti, 2016, p.90). En efecto, algunos autores aseguran que con la implementación de las NIIF mejora la calidad de la información financiera y por ende facilitará el Análisis de Estados Financieros, al mejorar la comparabilidad y transparencia (Janica \& Piñeiro, 2008; Karaibrahimoglu \& Tunç, 2014; García Carvajal \& Dueñas Casall, 2016).

Por otra parte, autores como Ball (2006) "difieren de tal perspectiva, al afirmar que las NIIF ignoran factores políticos y económicos profundamente arraigados que influyen en los incentivos de los preparadores de estados financieros y que inevitablemente conforman la práctica real de presentación de informes financieros". Por ende, debido a que los contextos institucionales difieren, es difícil acomodar la diversidad contable y lograr la armonización contable internacional. Los factores ambientales varían ampliamente a nivel mundial, pero juegan un papel importante en la explicación de las prácticas contables (Radebaugh, Gray \& Black, 2006). No obstante, Carmona y Trombetta (2008), sugieren "que el enfoque de los estándares basado en principios y su flexibilidad interna, permite la aplicación de NIC / NIIF a países con diversas tradiciones contables y condiciones institucionales variables" (p.455). Una característica distintiva de estos estándares es que se basan en principios en lugar de reglas (Carmona y Trombetta, 2008, p.456). Según Nelson (2003), "...las reglas incluyen criterios específicos, umbrales de 'línea brillante', ejemplos, restricciones de alcance, excepciones, precedentes posteriores, guía de implementación, etc. (p.91)". Por otro lado, los estándares "basados en principios" refieren a entendimientos fundamentales que informan transacciones y eventos económicos, y dominan cualquier otra regla establecida en el estándar (Carmona y Trombetta, 2008, p.456). En éste sentido, es importante señalar:

Es probable que un lenguaje de informes financieros globales eleve la comparabilidad internacional y el reconocimiento mundial de las PYME en todo el mundo. Por lo tanto, se espera que este cambio sea un gran avance para las PYMES si los beneficios percibidos son debidamente reconocidos por los países y adoptan las NIIF para las PYMES. (Perera \& Chand, 2015, p.165)

El IASB considera que la adopción de las NIIF para las PYME posiblemente mejorará el acceso de las PYME a las finanzas internacionales a través de información financiera armonizada y de alta calidad (IASB, 2009). Es así, que:

La necesidad de desarrollar un marco regulatorio apropiado y permitir que las PYME proporcionen información contable comparable se considera de gran importancia. Por lo tanto, es vital examinar las dificultades conceptuales y técnicas encontradas por aquellos países que se han apresurado a adoptar las NIIF para las PYMES. (Perera \& Chand, 2015, p.166)

El éxito de las NIIF se atribuye a sus estándares basados en principios (Carmona y Trombetta, 2008). Según Carneiro et al. (2017), "esta característica brinda flexibilidad a las NIIF y las ayuda a adaptarse a diferentes contextos institucionales y tradiciones contables. A pesar de la amplia adopción internacional de las NIIF, existen opiniones contradictorias sobre la calidad de los informes financieros" (p173).

Sin embargo, y debido a la publicación de NIIF para las PYMES y la desaceleración de la nueva norma por distintas jurisdicciones alrededor del mundo, las consecuencias de su adopción serán inminentes (Perera \& Chand, 2015, p.171). Carmona y Trombetta (2008) sostienen que la calidad de las NIIF depende de si la adopción es seria o si los países son "label adopters", es decir, buscan el prestigio reputacional de las NIIF, pero no se comprometen de forma innata y plena con ellas. Según Carneiro et al. (2017):

La armonización mediante la adopción de las NIIF tiene múltiples ventajas para las empresas multinacionales y los reguladores del mercado de capitales: reducción de costos, mejor comprensión, credibilidad y comparabilidad de la información financiera, y la oferta de una alternativa a los estándares establecidos por el Consejo de Normas de Contabilidad Financiera (FASB). (p.174)

Acorde con lo anterior, varias compañías han adoptado IFRS voluntariamente por razones competitivas y para obtener legitimidad (Guerreiro, Rodrigues, \& Craig, 2012). En general, estudios anteriores revelan que la implementación de las NIIF por países individuales parece ser un desafío, a pesar de que el estándar sea complejo o sea una versión simplificada. Tal es el caso de países como Argentina, Brasil, Chile, México y Perú, para los cuales a pesar de los esfuerzos para adoptar o converger con las NIIF, se presentaron diferencias significativas en la forma en que se aplicaron las NIIF, generando como resultado "estándares nacionales NIIF" (Sarquis, Luccas, \& Lourenço, 2014).

Por lo tanto, más investigación sobre la experiencia de transformación de informes financieros de y por entidades no responsables públicamente y PYMES es oportuna y esencial (Evans et al., 2005). Del mismo 
modo, la adopción de IAS / IFRS requiere que los contadores posean un sólido conocimiento de negocios y economía. "En este sentido, los contadores deben tener una comprensión integral de los fundamentos económicos y comerciales de las transacciones y eventos antes de decidir sobre su tratamiento contable" (Carmona y Trombetta, 2008, p.457).

\section{METODOLOGÍA}

La investigación analiza las implicaciones en el análisis financiero realizado bajo ratios financieros con la implementación de las NIIF para Pymes en Colombia. Para ello, se realizó un análisis pormenorizado del contenido de las NIIF para Pymes versión 2015 publicado por el International Accounting Standards Board (IASB) en conjunto con el marco normativo contable colombiano, contenido esencialmente en los Decretos 2649 y 2650 de 1993, y como soporte de éstos, la ley 43 de 1990, a fin de identificar los cambios sustanciales en los principios bajo los cuales se registran los hechos económicos que generan alteraciones en los ratios financieros, para cada uno de los elementos de la razón. También se tomó como soporte técnico los conceptos y pronunciamientos del Consejo Técnico de la Contaduría Pública, Portales Web dedicados al campo contable, financiero y tributario, y orientaciones de centros académicos. Los cambios identificados fueron debatidos con profesionales contables expertos en el tema a través de grupos de discusión, a fin de validar los hallazgos. Se elaboraron matrices para el registro de datos, que fueron el soporte para la organización de los resultados, las cuales contienen los argumentos de las variaciones en la razón, exponiendo por un lado los relacionados con aumentos y, por otro, con las disminuciones. Se escogieron los ratios financieros más representativos de la agrupación tradicionalmente conocida (liquidez, rotación, rentabilidad y endeudamiento) ${ }^{1}$. Como guía para el lector, se relacionan la(s) sección(es) de las NIIF que sustentan el respectivo argumento.

\section{RESULTADOS}

El análisis realizado evidencia diversos retos para los analistas financieros frente a la implementación del marco normativo contable internacional de las NIIF. En relación al Estado de Situación Financiera (antes llamado Balance General) desaparecen las cuentas de orden, cuyos rubros generalmente tienen impactos significativos en el Estado de Situación Financiera de Apertura (conversión). Necesariamente si los estados financieros sufren modificaciones, los ratios también se verán afectados, especialmente por la reclasificación de partidas y/o la inclusión de otras no consideradas en la norma contable colombiana como propiedades de inversión y equivalentes de efectivo y, por la exclusión de otras como las valorizaciones de activos y cargos diferidos. Esto coincide con otras investigaciones realizadas por los autores.

Los cambios en los principios bajo los cuales se registran los hechos económicos que generan alteraciones en los ratios financieros se describen en las tablas siguientes, según el tipo de indicador, comenzando por los ratios de liquidez, luego con los de rotación y rentabilidad, para finalmente terminar con los de endeudamiento. Es preciso aclarar que:

i. Dado que algunas razones para las variaciones se presentan en la convergencia o apertura (Estado de Situación Financiera de Apertura - ESFA) 2 .

ii. En razón de lo anterior, el análisis realizado a las diferentes secciones de las NIIF para Pymes, aplica en su mayoría para el proceso de implementación de dicha norma en las empresas colombianas; no siendo así para el proceso de convergencia o apertura, en razón de que la norma permite algunas excepciones, como se establece en la sección 35. 
Tabla 8

Variaciones en la rotación de activos fijos = ventas / activo fijo

Argumento de las variaciones en la razón

\section{Aumento Aumento de las ventas}

En el análisis realizado a las NIIF no se evidencian principios que originen un aumento de los ingresos (ventas) en comparación con los principios locales.

\section{Disminución activo fijo}

a. Por la medición del costo de partidas de propiedad, planta y equipo cuando los pagos se aplacen más allá de las condiciones normales de crédito - Valor presente $\neg-$ [S17.13, p.103].

b. Por el reconocimiento de los costos por préstamos como un gasto en resultados en el periodo en el que se incurre en ellos, en vez de capitalizarlos [S25.2, p.168]

c. Por la distribución del costo inicial del activo y depreciación de cada uno de estos componentes por separado a lo largo de su vida útil, lo que cambia el valor neto de las partidas de propiedad, planta y equipo [S17.16, p.104]

d. Cambio en el patrón de desgaste, de depreciación en línea recta a desgaste acelerado en los primeros años, genera alta rotación debido a menor nivel de activos. (Van Greunning, 2010, p.71) [S17.23, p.105-106]

e. Por la clasificación de elementos de propiedad, planta y equipo como propiedad de inversión, según la intención que se tenga para el activo. Esto es completamente nuevo con respecto a la norma contable colombiana [S16.3, p.98]

f. Disminución en el valor de salvamento y en la vida depreciable del activo, genera alta rotación debido a menor nivel de activos. (Van Greunning, 2010, p.71) [S17.19, p.105]

\section{Disminución Disminución de las ventas}

a. Por operaciones comerciales que impliquen una transacción financiera, se deberán medir al valor razonable de todos los cobros futuros determinados utilizando una tasa de interés imputada [S23.5, p. 151].

b. Por la no satisfacción de las cinco condiciones establecidas para el reconocimiento de las ventas de bienes [S23.10-11-12-13, p.152], en cuyo caso no se debe reconocer el ingreso en cuentas hasta que total cumplimiento de las mismas, situación que no se contemplaba en las normas contables colombianas.

Por la aplicación del método del porcentaje de terminación

c. aplicado a la prestación de servicios y contratos de construcción en la estimación de los ingresos de acuerdo al grado de terminación de los mismos [S23.14 a S23.27, p.155].

Por la aplicación de descuentos por pronto pago [S23.3, p.150], dado

d. que bajo NIIF se debe reconocer el valor del descuento inmediatamente (momento de la venta), incluso si es incierta la aplicación del descuento.

\section{Aumento activo fijo}

a. Por la distribución del costo inicial del activo y depreciación de cada uno de estos componentes por separado a lo largo de su vida útil, lo que cambia el valor neto de las partidas de propiedad, planta y equipo [S17.16, p.104]

b. Reconocimiento de piezas de repuesto, equipo de reserva y el equipo auxiliar como propiedades, planta y equipo. [S17.5, p.101]

c. ${ }^{*}$ Por el reconocimiento de activos no incluidos en la contabilidad [S35.5, p.236].

d. *Por la valuación de partidas de propiedad, planta y equipo, propiedades de inversión e intangibles en el ESFA [S35.9(c), p.237]. Por la inclusión en los componentes del costo de partidas de propiedad, planta y equipo como costos de desmantelamiento, retiro de elementos y rehabilitación del lugar [S17.10(c), p.102].

Fuente: Elaboración propia

Tabla 9

Variaciones en el rendimiento de activos $=$ utilidad neta / activo total

Argumento de las variaciones en la razón

\section{Aumento Aumento de la utilidad neta}

a. Por la disminución de los cargos por depreciación del periodo causados por cambios en las estimaciones de vidas útiles de activos de propiedad, planta y equipos [S17.22, p.105].

b. *Por la disminución de los cargos por depreciación del periodo causados por el reconocimiento de partidas de propiedad, planta y equipo como propiedades de inversión medidas al valor razonable, las cuales no se deprecian [16.7, p.99].

c. Por las subvenciones no condicionadas recibidas, que se registran como ingresos de actividades ordinarias cuando los importes obtenidos por la subvención sean exigibles. [S24.4(a), p. 166]

\section{Disminución del activo total}

a. Por la medición del costo de partidas de propiedad, planta y equipo cuando los pagos se aplacen más allá de las condiciones normales de crédito - Valor presente ᄀ- [S17.13,p.103].

b. *Las NIIF no contemplan partidas relacionadas con valorizaciones de activos realizadas luego del ESFA, lo cual se permitía con las normas contables colombiana, donde se registraban en el grupo de cuentas denominado "Valorizaciones" [35.8, p.236]

c. *Las NIIF no contemplan el reconocimiento de partidas intangibles registradas como formados o creados según estimaciones realizadas bajo el marco contable colombiano [S18.4C, p.109; S18.14, p.110; S18.17, p.111; FC114]

d. *Las NIIF no contemplan el reconocimiento de partidas capitalizadas como activos tales como gastos o cargos diferidos - preoperativos, investigaciones, mejoras, etc. - [S18.15, p.111].

e. Por la restricción en el uso del efectivo o equivalentes al efectivo. [S4.5(d), p.31]

\section{Disminución Disminución de la utilidad neta}

a. Por la aplicación de descuentos por pronto pago [S23.3, p.150], dado que bajo NIIF se debe reconocer el valor del descuento inmediatamente (momento de la venta), incluso si es incierta la aplicación del descuento.

b. Por operaciones comerciales que impliquen una transacción financiera, se deberán medir al valor razonable de todos los cobros futuros determinados utilizando una tasa de interés imputada [S23.5, p. 151].

c. Por la no satisfacción de las cinco condiciones establecidas para el reconocimiento de las ventas de bienes [S23.10-11-12-13, p.152], en cuyo caso no se debe reconocer el ingreso en cuentas hasta que total cumplimiento de las mismas, situación que no se contemplaba en las normas contables colombianas.

d. Por la aplicación del método del porcentaje de terminación aplicado a la prestación de servicios y contratos de construcción en la estimación de los ingresos de acuerdo al grado de terminación de los mismos [S23.14 a S23.27, p.155].

e. Por los gastos incurridos en partidas intangibles cuando las mismas no cumplan con los criterios para el reconocimiento de activos [S18.14, p.109].

\section{Aumento del activo total}

a. ${ }^{*}$ Por el reconocimiento de activos no incluidos en la contabilidad [S35.5, p.236].

b. *Por la valuación de partidas de propiedad, planta y equipo, propiedades de inversión e intangibles en el ESFA [S35.9(c), p.237]

c. Por la medición de los inventarios al valor neto de realización [S13.4, p.84] 
Tabla 9

\section{Variaciones en el rendimiento de activos $=$ utilidad neta $/$ activo total}

\section{Disminución del activo total}

f. Cuando la medición de inventarios resulte inferior al costo - precio de venta estimado menos los costos de terminación y venta [S13.4, p.84]

g. *Baja en cuentas de activos financieros. [..] una entidad tendrá la opción de elegir entre (a) darlos de baja en el momento de la adopción de la NIIF para las PYMES; o (b) seguir reconociéndolos hasta que se proceda a su disposición o hasta que se liquiden. [S35.9(a), p.236].

h. Por el reconocimiento de valor residual cero de un activo intangible. [18.23, p.112]

i. Revisión del periodo y del método de amortización de intangibles, por un cambio en el uso, avances tecnológicos o cambios en los precios de mercado [S18.24, p.112].

\section{Aumento del activo total}

d. Por la inclusión en los componentes del costo de partidas de propiedad, planta y equipo como costos de desmantelamiento, retiro de elementos y rehabilitación del lugar donde operaron los activos [S17.10(c), p.102].

e. Por la medición posterior de partidas de propiedad, planta y equipo medidas al costo revaluado [S17.15, p.103].

f. Cuando las operaciones constituyan una transacción de financiación, se medirá el activo financiero al valor presente de los pagos futuros descontados a una tasa de interés de mercado para un instrumento de deuda similar determinado en el reconocimiento inicial. [S11.13, p.63].

Fuente: Elaboración propia

Tabla 10

Variaciones en Rendimiento sobre el Patrimonio = Utilidad Neta / Patrimonio

Argumento de las variaciones en la razón

\section{Aumento Aumento de la Utilidad Neta \\ a. Disminución en el valor de salvamento y en la vida depreciable del activo, genera alta rotación debido a menor nivel de activos. (Van Greunning, 2010, p.71) [S17.19-22, p.105] \\ b. Por el reconocimiento de las propiedades de inversión. [S16.2, p.98] \\ c. *Por la disminución de los cargos por depreciación del periodo causados por el reconocimiento de partidas de propiedad, planta y equipo como propiedades de inversión medidas al valor razonable, las cuales no se deprecian [16.7, p.99].}

\section{Disminución del Patrimonio}

a. Medición inicial de instrumentos de patrimonio, sobre la base del valor presente. [S22.8, p.143]

\section{Disminución Disminución de la Utilidad Neta}

a. Reconocimiento como gastos de costos excluidos de un elemento de propiedades, planta y equipo. [S17.11, p.103]

b. Cambio en el patrón de desgaste, de depreciación en línea recta a desgaste acelerado en los primeros años, genera alta rotación debido a menor nivel de activos. (Van Greunning, 2010, p.71) [S17.23, p.105-106]

c. Una entidad reconocerá todos los costos por préstamos como un gasto en resultados en el periodo en el que se incurre en ellos. [S25.2, p.168]

d. Reconocimiento de piezas de repuesto, equipo de reserva y el equipo auxiliar como propiedades, planta y equipo. [S17.5, p.101]

e. Por los gastos incurridos en partidas intangibles cuando las mismas no cumplan con los criterios para el reconocimiento de activos [S18.14, p.109].

f. Por la aplicación de descuentos por pronto pago [S23.3, p.150], dado que bajo NIIF se debe reconocer el valor del descuento inmediatamente (momento de la venta), incluso si es incierta la aplicación del descuento.

g. Por operaciones comerciales que impliquen una transacción financiera, se deberán medir al valor razonable de todos los cobros futuros determinados utilizando una tasa de interés imputada [S23.5, p. 151].

h. Por la no satisfacción de las cinco condiciones establecidas para el reconocimiento de las ventas de bienes [S23.10-11-12-13, p.152], en cuyo caso no se debe reconocer el ingreso en cuentas hasta que total cumplimiento de las mismas, situación que no se contemplaba en las normas contables colombianas.

i. Por la aplicación del método del porcentaje de terminación aplicado a la prestación de servicios y contratos de construcción en la estimación de los ingresos de acuerdo al grado de terminación de los mismos [S23.14 a S23.27, p.155].

\section{Aumento del Patrimonio}

a. Clasificación de un instrumento financiero como un patrimonio [S22.4, p.141]

b. Aportaciones de socios de cooperativas reconocidas como patrimonio. [S22.6, p.142]

c. Por el acuerdo de distribución de dividendos a los tenedores de sus instrumentos de patrimonio después del final del periodo sobre el que se informa. [S32.8, p.222] 
Tabla 11

Variaciones en Leverage Total $=$ Pasivo Total $/$ Patrimonio

\section{Aumento Aumento del Pasivo Total}

a. Costo de propiedades, planta y equipo. Estimación inicial de los costos de desmantelamiento o retiro del elemento. [S17.10, p.102]

b. Clasificación de un instrumento financiero como un pasivo financiero [S22.3A; 22.5, p.141-142]

c. Por las subvenciones recibidas antes de que satisfagan los criterios de reconocimiento de ingresos de actividades ordinarias. [S24.4(b, c), p.166]

d. Hechos ocurridos después del periodo sobre el que se informa que implican ajuste. [S32.5(a), p.220]

e. Por el reconocimiento de pasivos contingentes en una combinación de negocios. [S2.40, p.21; S19.15(d), p.117; S19.20, p.118]

\section{Disminución del Patrimonio}

b. Medición inicial de instrumentos de patrimonio, sobre la base del valor presente. [S22.8, p.143]

Fuente: Elaboración propia

\section{Disminución Disminución del Pasivo Total}

a. Por la medición del pasivo por beneficios definidos. [S28.15, p.190; 28.17, p.191]

b. Por el no reconocimiento de pasivos cuando se acuerda distribuir dividendos después del final del periodo sobre el que se informa. [S32.8, p.222]

c. Por el no reconocimiento como pasivos, de partidas que cumplan con la definición de pasivos. [S2.16, p.16; S2.27, p.19; S2.45, p.22]

d. Por la medición al costo amortizado utilizando el método del interés, de los instrumentos de deuda. [S11.13, p.63; S11.14, p.64; S11.15, p.65]

e. Por el no reconocimiento de partidas como pasivos, no permitidos por las NIIF para Pymes. [S35.7(b), p.236]

\section{Aumento del Patrimonio}

d. Clasificación de un instrumento financiero como un patrimonio [S22.4, p.141]

e. Aportaciones de socios de cooperativas reconocidas como patrimonio. [S22.6, p.142]

f. Por el acuerdo de distribución de dividendos a los tenedores de sus instrumentos de patrimonio después del final del periodo sobre el que se informa. [S32.8, p.222]

Tabla 12

Variaciones en Cobertura de Intereses = Utilidad Antes de Intereses e Impuestos [UAII] $/$ Gastos Financieros

Argumento de las variaciones en la razón

\section{Aumento Aumento de la UAII}

a. Disminución en el valor de salvamento y en la vida depreciable del activo, genera alta rotación debido a menor nivel de activos. (Van Greunning, 2010, p.71) [S17.19-22, p.105]

b. Por el reconocimiento de las propiedades de inversión. [S16.2, p.98]

c. ${ }^{*}$ Por la disminución de los cargos por depreciación del periodo causados por el reconocimiento de partidas de propiedad, planta y equipo como propiedades de inversión medidas al valor razonable, las cuales no se deprecian [16.7, p.99].

d. Por las subvenciones no condicionadas recibidas, que se registran como ingresos de actividades ordinarias cuando los importes obtenidos por la subvención sean exigibles. [S24.4(a), p. 166]

e. Por la presentación de partidas de gasto reconocidas en el resultado integral. [S5.2, p.34]

\section{Disminución de Gastos Financieros}

a. Clasificación de un instrumento financiero como un patrimonio [S22.4, p.141]

b. *Baja en cuentas de un pasivo financiero [S11.36, p.73]

c. *Baja en cuentas de pasivos financieros. [..] una entidad tendrá la opción de elegir entre (a) darlos de baja en el momento de la adopción de la NIIF para las PYMES; o (b) seguir reconociéndolos hasta que se proceda a su disposición o hasta que se liquiden. [S35.9(a), p.236].

\section{Disminución Disminución de la UAII}

a. Por el no reconocimiento de activos cuando no sea probable que de los mismos se vaya a obtener beneficios económicos futuros. [S2.37, p.21]

b. Por la presentación de partidas de ingreso reconocidas en el resultado integral. [S5.2, p.34]

c. Reconocimiento como gastos de costos excluidos de un elemento de propiedades, planta y equipo. [S17.11, p.103]

d. Cambio en el patrón de desgaste, de depreciación en línea recta a desgaste acelerado en los primeros años, genera alta rotación debido a menor nivel de activos. (Van Greunning, 2010, p.71) [S17.23, p.105-106]

e. Una entidad reconocerá todos los costos por préstamos como un gasto en resultados en el periodo en el que se incurre en ellos. [S25.2, p.168]

f. Reconocimiento de piezas de repuesto, equipo de reserva y el equipo auxiliar como propiedades, planta y equipo. [S17.5, p.101]

g. Por los gastos incurridos en partidas intangibles cuando las mismas no cumplan con los criterios para el reconocimiento de activos [S18.14, p.109].

h. Por la aplicación de descuentos por pronto pago [S23.3, p.150], dado que bajo NIIF se debe reconocer el valor del descuento inmediatamente (momento de la venta), incluso si es incierta la aplicación del descuento.

i. Por operaciones comerciales que impliquen una transacción financiera, se deberán medir al valor razonable de todos los cobros futuros determinados utilizando una tasa de interés imputada [S23.5, p. 151].

j. Por la no satisfacción de las cinco condiciones establecidas para el reconocimiento de las ventas de bienes [S23.10-11-12-13, p.152], en cuyo caso no se debe reconocer el ingreso en cuentas hasta que total cumplimiento de las mismas, situación que no se contemplaba en las normas contables colombianas.

k. Por la aplicación del método del porcentaje de terminación aplicado a la prestación de servicios y contratos de construcción en la estimación de los ingresos de acuerdo al grado de terminación de los mismos [S23.14 a S23.27, p.155].

l. Por el reconocimiento como gastos del periodo de los costos indirectos no distribuidos. [S13.9, p.85] 
Tabla 12

Variaciones en Cobertura de Intereses = Utilidad Antes de Intereses e Impuestos [UAII] / Gastos Financieros

Argumento de las variaciones en la razón

Aumento de Gastos Financieros

a. Por los instrumentos de deuda que cumplan las condiciones del párrafo 11.8(b), que se medirán al costo amortizado utilizando el método del interés efectivo. [S11.14-15, p.65]

b. Por el reconocimiento de los costos por préstamos como un gasto en resultados en el periodo en el que se incurre en ellos, en vez de capitalizarlos [S25.2, p.168].

c. Por la medición posterior de los instrumentos financieros. [S11.14-16, p.64-65]

d. Por el reconocimiento de gastos por intereses al adquirir inventarios con pago aplazado. [S13.7, p.85]

Fuente: Elaboración propia

\section{DISCUSIÓN}

La implementación de las NIIF en Colombia trajo consigo profundos cambios en algunos principios que sustentan la forma de reconocer y medir hechos económicos, además de retornar la vocación de la contabilidad al campo de las finanzas, consolidando una relación que las leyes y costumbres colombianas menguaron en beneficio de normas tributarias. En este sentido, por ejemplo, se exige la aplicación de un principio fundamental en las finanzas (Keown, Martin, \& Petty, 2014): el dinero tiene un valor en el tiempo, que la contabilidad debe reconocer y evidenciar en los registros en los cuales se hace necesario. Ello va en detrimento de algunas disposiciones tributarias, pero se hace imprescindible en el objetivo contable que busca la representación fiel de la realidad económica, y lo cierto es que el dinero tiene un valor en el tiempo. Con el método de interés efectivo se busca facilitar los cálculos para el reconocimiento contable de hechos económicos que implican operaciones de crédito con interés explícito o implícito (transacción de financiación).

Con el análisis se evidencia que los cambios en los resultados de aplicación de ratios financieros tendrán diversos orígenes, que en ocasiones pueden llegar a afectar a un mismo indicador en varias ocasiones, ante lo cual el analista financiero deberá enfocar su atención en detectar las causas que realmente provocan las variaciones.

En este sentido, los analistas financieros deberán entender los nuevos principios bajo los cuales se prepara la contabilidad, adaptándose incluso a un nuevo lenguaje técnico-contable, originado por nuevas partidas en los estados financieros o el cambio en el nombre de otras ya existentes en la contabilidad local.

Por otra parte, la implementación de las NIIF en Pymes implica una oportunidad para que éstas puedan interactuar de mejor manera frente a las necesidades empresariales e informativas de los mercados internacionales. Así, el análisis financiero se fortalece como herramienta para la toma de decisiones al manejar un lenguaje común con firmas de otros países. Contrario a esto, también es necesario tener presente que la experiencia de algunos expertos indica que las NIIF, al basarse en principios, puede llegar a ser laxa o permisiva en algunos aspectos, permitiendo a las empresas escoger políticas contables que necesariamente inciden en la presentación de la información financiera y, por ende, en el análisis financiero.

\section{CONCLUSIONES}

Necesariamente con la implementación de las NIIF para Pymes se afectan los ratios financieros, en razón de que algunos de los principios de contabilidad contenidos en el marco normativo contable internacional cambian sustancialmente en relación a las normas contables locales. Algunos efectos sólo se evidencian en la convergencia a NIIF (ESFA), mientras otros se presentan con la implementación (primer año en adelante), dado que la sección 35 permite algunas excepciones. Esto obliga a los analistas financieros a identificar con exhaustividad el origen de cambios que sufran los indicadores financieros de empresas, que pueden mostrar deterioros sin que ello sea causado por razones operacionales sino por los ajustes en la forma de llevar la contabilidad. Ejemplo de ello es el deterioro del valor de los activos, conocido localmente como provisión, el cual se realiza bajo los parámetros de las NIIF de forma más estructurada atendiendo a la esencia de la realidad económica y no a la forma legal.

Otro caso se presenta con la reclasificación presentada en algunos elementos de los estados financieros, como el reconocimiento de activos de propiedad, planta y equipo como propiedad de inversión, tópico no conocido en la norma contable colombiana, o la estimación de los costos de desmantelamiento, retiro de elementos y rehabilitación del lugar donde operaron activos como maquinaria y equipo industrial que pueden llegar a afectar el medioambiente. Igualmente pasa con el afianzamiento de la relación contabilidad-finanzas, que perdió fuerza con las normas contables locales, orientadas más a requerimientos 
normativo-tributarios. Ejemplo de ello es la aplicación del método de interés efectivo, que implica soportar operaciones de crédito que se extiendan más allá de los plazos normales bajo el principio del valor del dinero en el tiempo, pilar fundamental de las finanzas.

Dado los resultados de la presente investigación, se hace necesario realizar futuros estudios que impliquen la construcción de nuevos ratios financieros que miden el efecto de las nuevas partidas que se incluyen en los estados financieros, como por ejemplo, el Otro Resultado Integral (ORI).

\section{REFERENCIAS BIBLIOGRAFICAS}

Alp, A., \& Ustundag, S. (2009). Financial reporting transformation: The experience of Turkey. Critical . Perspectives on Accounting, 20(5), 680-699.

Álvarez, B., Orozco, E., \& León, G. (2013). Las NIIF y su impacto en las cooperativas en Colombia a diciembre 31 de 2011. Cuadernos de Administración, 29(50), 176-185.

Arango, D., \& Torres, A. (2014). Análisis del proceso de armonización de las normas internacionales de contabilidad e información financiera (NIIF-NICSP) en el sector público en Colombia. Contexto: Revista de la Facultad de Ciencias Económicas Administrativas y Contables, 3(1), 132-145. Obtenido de http://bibliotecadigital.usb.edu.co:2072/ehost/pdfviewer/ pdfviewer?sid=82bb08a0-dfb1-4a12-b681-e9c068246e89\%40sessionmgr4007\&vid=0\&hid=4114

Ball, R. (2006). International Financial Reporting Standards (IFRS): pros and cons for investors. Accounting and Business Research, 36(1), 5-27. doi:10.1080/00014788.2006.9730040

Cagnazzo, L., Tiacci, L., Cardoni, A., \& Brilli, M. (2012). Enterprises, PRO-VE 2012 - IFIP Advances in Information and Communication Technology 380, 503-512. doi:10.1007/978-3-662-44745-1_29

Carmona, S., \& Trombetta, C. (2008). On the global acceptance of IAS/IFRS accounting standards: The logic and implications of the principlesbased system. Journal of Accounting and Public Policy, 27, 455-461. doi:10.1016/j.jaccpubpol.2008.09.003

Carneiro, J., Rodrigues, L. L., \& Craig, R. (2017). Assessing international accounting harmonization in Latin America. Accounting Forum, 41, 172-184. doi:10.1016/j.accfor.2017.06.001

Castellanos-Sánchez, H. A. (2015). Medición de activos no financieros. Un análisis de los elementos que intervienen en la decisión de la gerencia al optar por el modelo del valor razonable. Cuadernos de Contabilidad, 16(40), 41-71. Obtenido de dx.doi.org/ 10.11144/Javeriana.cc16-40.manf

Chen, J. (2006). Development of Chinese small and medium-sized enterprises. Journal of Small Business and Enterprise Development, 13(2), 140-147.

Choi, F., \& Meek , G. (2011). International accounting (7th ed.). New Jersey, United States of America: Prentice Hall. Pearson Education.

Confecámaras - Red de Cámaras de Comercio. (2015). Impacto del sistema general de regalías en el sector productivo priorizado por las comisiones regionales de competitividad. Bogotá DC.

Confetti, R., Da Silva, J., Ambrozini, M., \& Guasti, F. (2016). Impact of adopting IFRS standard on the equity cost of brazilian open capital companies. Revista de Administração Mackenzie, 17(4), 85-108. doi:10.1590/1678-69712016/administracao.v17n4p84-107

Deloitte. (2013). Generalidades de las NIIF. Sensibilización al entorno IFRS / NIIF. Deloitte ToucheTohmatsu.

Entwistle, G. (2015). Reflections on Teaching Financial Statement Analysis. Accounting Education an international journal, 24, 555-558. doi:10.1080/09639284.2015.1075314

Evans, L., Gebhardt, G., Hoogendoorn, M., Marton, J., Di Pietra, R., Mora, A.,... Wagenhofer, A. (s.f.). Problems and opportunities of an international financial reporting standard for small and medium-sized entities: The EAA FRSC's comment on the IASB's discussion paper. Accounting in Europe, 2(1), 23-45.

Feroz, E., Kim, S., \& Raab, R. (2003). Financial statement analysis: A data envelopment analysis approach. Journal of the Operational Research Society, 54, 48-58. Obtenido de http://ezproxy.uninorte.edu.co:2124/article/10.1057/palgrave.jors.2601475

García Carvajal, S., \& Dueñas Casall, R. (2016). Los aspectos cualitativos en las Pymes y los nuevos retos gerenciales frente a las NIIF. Revista Academia \& Virtualidad, 9(2), 108-120. doi:http://dx.doi.org/10.18359/ravi.2010

García Padilla, V. M. (2015). Análisis financiero. Un enfoque integral. D.F., México: Grupo Editorial Paria.

Guajardo Cantú, G., \& Andrade de Guajardo, N. E. (2008). Contabilidad financiera (5 ed.). D.F., México: Mc Graw Hill.

Guerreiro, M. S., Rodrigues, L. L., \& Craig, R. (2012). Voluntary adoption of International Financial Reporting Standards by large unlisted companies in Portugal: Institutional logics and strategic responses. Accounting, Organizations and Society, 37, $482-499$.

Guevara Sanabria, J. A., \& Correa García, J. A. (2014). Impactos de la información e indicadores financieros, por la convergencia a NIIF plenas: propiedad, planta y equipo. XIV Asamblea general de ALAFEC. ALAFEC. Ciudad de Panamá: Asociación Latinoamericana de Facultades y Escuelas de Contaduría y Administración. Obtenido de http://www.alafec.unam.mx/docs/asambleas/xiv/ ponencias/2.09.pdf

Horngren, C. T., Harrison Jr., W., \& Oliver, M. (2010). Contabilidad (8 ed.). D.F., México: Pearson Educación.

IFRS. (2017). Analysis of the IFRS jurisdiction profiles. Obtenido de http://www.ifrs.org/use-around-the-world/use-of-ifrs-standards-by-jurisdiction /\#analysis

INSTITUTO DE CONTABILIDAD Y AUDITORÍA DE CUENTAS (ICAC). (2002). Informe sobre la situación actual de la contabilidad en España y líneas básicas para abordar su reforma. Madrid: ICAC. 
International Accounting Standards Board (IASB). (2009). International financial reporting standards (IFRS) for small and medium sized entities (SME). Obtenido de http://www.ifrs.org/ifrs-for-smes/Pages/ifrs-for-smes.aspx

Janica , F., \& Piñeiro, J. (2008). USGAAP vs, IFRS y COLGAAP Lo Básico. Ernst \&Young Audit Ltda. Obtenido de www.globalcontable.com/archivos generales/secciones/ifrs/comparativos/ey/colgaapbasico.pdf.

Karaibrahimoglu, Y. Z., \& Tunç, G. (2014). Financial Statement Analysis under IFRS. En N. Ray, \& K. Chakraborty, Handbook of Research on Strategic Business Infrastructure Development and Contemporary Issues in Finance (págs. 238-255). United States of America: IG Global). doi:10.4018/978-1-4666-5154-8.ch017

Keown, A., Martin, J., \& Petty, W. (2014). Foundations of Finance (8 ed.). New Jersey, United States of America: Pearson.

Lev, B. (1971). Financial Failure and Informational Decomposition Measures. Accounting in perspective : contributions to accounting thought by other disciplines, 102-111.

LEY 1314 DE 2009. (13 de julio de 2009). Obtenido de http://www.alcaldiabogota.gov.co/sisjur/normas/Norma1.jsp?i=36833

Ley 905 de 2004. (2 de Agosto de 2004). Obtenido de http://www.secretariasenado.gov.co/senado/basedoc/ley_0905_2004.html

Mayes, T. R., \& Shank, T. M. (2012). Financial analysis with Microsoft Excel (6th ed.). United States of America: Cengage Learning.

Meigs, R., Williams, J., Haka, S. F., \& Bettner, M. S. (2000). Contabilidad. La base para decisions gerenciales (11 ed.). Bogotá, Colombia: Irwin Mc Graw Hill.

Molina, H., \& Tua, J. (2010). Reglas versus Principios contables, ¿son modelos incompatibles? Revista española de financiación y contabilidad, XXXIX(146), 259-287.

Muñoz Jiménez, J. (2008). Contabilidad financiera. Madrid, España: Pearson Prentice Hall.

Nelson, M. W. (2003). Behavioral evidence on the effects of principles- and rules-based standards (commentary). Accounting Horizons, 17(1), 91-104. doi:10.2308/acch.2003.17.1.91

Olave, J. C. (27 de febrero de 2013). La adopción de las NIIF en Colombia. Portafolio. Obtenido de http://www.portafolio.co/opinion/juan-carlosolave/adopcion-niif-colombia-76182

Organization for Economic Co-operation and Development (OECD). (2005). OECD SME and entrepreneurship outlook. OECD Publication Service. Obtenido de http://www.oecd.org/cfe/smes/oecdsmeandentrepreneurshipoutlook-2005edition.htm

Özkan, S., \& Erdener, E. (2010). Uluslararası muhasebe/finansal raporlama standartlarinin finansal tablolar analizi üzerindeki etkilerine genel bakiş. . Mali Çözüm, 97, 49-86. Obtenido de http://archive.ismmmo.org.tr/docs/malicozum/97malicozum/3\%20serdar\%20ozkan.pdf

Perera, D., \& Chand, P. (2015). Issues in the adoption of international financial reporting standards (IFRS) for small and medium-sized enterprises (SMES). Advances in Accounting. Incorporating Advances in International Accounting, 31, 165-178. doi:10.1016/j.adiac.2015.03.012

Perera, D., \& Chand, P. (2015). Issues in the adoption of international financial reporting standards (IFRS) for small and medium-sized enterprises (SMES). Advances in Accounting, Incorporating Advances in International Accounting, 31, 165-178. doi:10.1016/j.adiac.2015.03.012

Puerta, F., Vergara, J., \& Portela, L. (2017). Propuesta de ajuste en los ratios financieros tradicionales a partir del análisis de las Normas Internacionales de Información Financiera para Pymes - NIIF para Pymes: Caso Colombia. Memorias Simposio de Investigación 2017 (págs. 218-229). Cartagena: Editorial Universidad Tecnológica de Bolívar.

Radebaugh, L., Gray, S., \& Black, E. (2006). International accounting and multinational enterprises. New York, United States of America: Wiley

Reddy, M. (2007). Small business in small economies: Constraints and opportunities for growth. Social and Economics Studies, 56(1/2), 304-321.

Rees, B. (1991). Financial analysis. USA: Prentice Hall

Robinson, T. R., Van Greuning, H., Henry, E., \& Broihahn, M. A. (2009). International Financial Statement Analysis. Hoboken, New Jersey, United States of America: CFA Institute.

RSM International Association. (2009). A guide through IFRS for SMEs. Obtenido de http://www.rsmi.com/attachments/approved/rsminternational-service-literature/en/FINALIFRSforSMEs.pdf

Salazar, É. (2011). Análisis de las implicaciones no financieras de la aplicación de la NIIF para PYME en las medianas entidades en Colombia. Cuadernos de Contabilidad, 12(30), 211-241

Salazar, E. (2013). Efectos de la implementación de la NIIF para las PYMES en una mediana empresa ubicada en la ciudad de Bogotá. Cuadernos de Contabilidad, 14(35), 395-414

Sánchez Fernández de Valderrama, J. L. (2006). Las consecuencias de las NIC/NIIF para el Análisis Financiero . Madrid: academia.edu. Obtenido de http://s3.amazonaws.com/academia.edu.documents/34899007/01_consecuencias.pdf?AWSAccessKeyId=AKIAIWOWYYG

Sarquis, R. W., Luccas, R. G., \& Lourenço, I. (2014). Classificação dos Sistemas Contábeis na era IFRS: uma análise dos países da América Latina. XIV Congresso USP de Controladoria e Contabilidade. São Paulo.

Sava, R., Mârza, B., \& Eşanu, N. (2013). Financial Reporting for SMEs Past and Perspectives. Procedia Economics and Finance, 6, 713 - 718.

Van Horne, J. C., \& Machowicz, J. M. (2010). Fundamentos de Aministraciòn Financiera (13 ed.). Naucalpan de Juárez, México: Pearson.

Warren, C. S., Reeve, J. M., \& Duchac, J. E. (2010). Contabilidad financiera (11 ed.). D.F., México: Cengage learning.

Wild, J. J., Subramanyam, K. R., \& Halsey, R. F. (2007). Análisis de estados financieros (9ºd.). D.F., México: McGraw-Hill.

Wong , P. (2004). Challenges and successes in implementing international: standardsachieving convergence to IFRSs and IASs. . International Federation of Accountants. 